\section{Disforia de Gênero em crianças: revisão integrativa da literatura e recomendações para o manejo na Atenção Primária à Saúde}

\author{
Gender Dysphoria in children: integrative literature review and \\ management recommendations in Primary Health Care
}

Disforia de Género en niños: revisión integrativa de la literatura y recomendaciones para el manejo en la Atención Primaria a la Salud

\section{Resumo}

Introdução: Disforia de Gênero em crianças é um tema pouco abordado no treinamento clínico, apesar do aumento do interesse das famílias sobre as questões de gênero. Objetivos: Realizar revisão integrativa de pesquisas sobre Disforia de Gênero em crianças e identificar recomendações para o manejo na Atenção Primária à Saúde. Métodos: Revisão integrativa da literatura utilizando termos MeSH nas bases de dados Pubmed, Medline, Lilacs e Scielo para artigos publicados entre 2008 e 2018 que utilizaram crianças transgêneras em suas análises. Resultados: Dos 2.488 artigos identificados pela chave de busca, 12 artigos foram selecionados para estudo. A maioria de centros especializados no atendimento a crianças transgêneras em quatro países. O profissional de saúde deve realizar anamnese direcionada às questões de comportamento da criança em casa e na escola, dinâmica familiar, contexto cultural, história familiar de não conformidade de gênero, vida social da criança e segurança infantil. Deve-se atentar para a avaliação psicossocial adequada para a idade da criança. Cabe à equipe de Atenção Primária à Saúde esclarecer as principais dúvidas das famílias, realizar a suspeita diagnóstica e encaminhar ao centro especializado no atendimento a crianças transgêneras do seu estado. Conclusão: Cabe à equipe de Atenção Primária à Saúde providenciar um ambiente acolhedor e sem discriminação para que estas famílias sejam avaliadas e conduzidas de forma adequada.

Palavras-chave: Identidade de Gênero; Saúde e Gênero; Pessoas Transgênero; Disforia de Gênero; Criança

Como citar: Mariano TSO, Moretti-Pires RO. Disforia de Gênero em crianças: revisão integrativa da literatura e recomendações para o manejo na Atenção Primária à Saúde. Rev Bras Med Fam Comunidade. 2018;13(40):1-11. http://dx.doi.org/10.5712/rbmfc13(40)1653
Tatiana da Silva Oliveira Mariano, Rodrigo Otávio Moretti-Pires

Universidade Federal de Santa Catarina (UFSC). Florianópolis, Santa Catarina, SC, Brasil.

oliveira.tatianasilva@gmail.com

(Autora correspondente); rodrigo.moretti@ufsc.br

Fonte de financiamento: declaram não haver. Parecer CEP: não se aplica. Conflito de interesses: declaram não haver. Procedência e revisão por pares: revisado por pares. Recebido em: 10/11/2017. Aprovado em: 24/12/2018. 


\begin{abstract}
Introduction: Gender dysphoria in children is a subject rarely addressed in clinical training, despite the increased interest of families on gender issues. Objective: Achieve an integrative review of papers about Gender Dysphoria in children and identify recommendations for management in Primary Health Care. Methods: Integrative literature review using MeSH terms in the Pubmed, Medline, Lilacs and Scielo databases for articles published between 2008 and 2018 that used transgender children in their analyzes. Results: From the 2,488 articles identified by the search key, 12 articles were selected. Most of them are from specialized centers for the care of transgender children in four countries. The health professional should carry out anamnesis directed to the child's behavioral issues at home and at school, family dynamics, cultural context, family history of non-compliance of gender, social life of the child and child safety. Attention should be paid to psychosocial assessment appropriate to the child's age. It is up to the Primary Health Care team to clarify the main doubts of the families, to carry out the diagnostic suspicion and to refer to the specialized center for the care of the transgender children of their state. Conclusion: It is up to the Primary Health Care team to provide a welcoming and non-discriminatory environment so these families can be properly evaluated and conducted.
\end{abstract}

Keywords: Gender Identity; Gender and Health; Transgender Persons; Gender Dysphoria; Child

\title{
Resumen
}

Introducción: La disforia de género en los niños es un tema poco abordado en el entrenamiento clínico, a pesar del aumento del interés de las familias sobre las cuestiones de género. Objetivo: Lograr una revisión integrativa de investigación sobre la Disforia de Género en los niños e identificar recomendaciones para el manejo en Atención Primaria de Salud. Métodos: Revisión integrativa de la literatura utilizando términos MeSH en las bases de datos Pubmed, Medline, Lilacs y Scielo para artículos publicados entre 2008 y 2018 que utilizaron niños transgéneros en sus análisis. Resultados: De los 2.488 artículos identificados por la clave de búsqueda, 12 artículos fueron seleccionados. En su mayoría, de centros especializados en atención a los niños transgéneros de cuatro países. El profesional de salud debe realizar anamnesis dirigida a las cuestiones de comportamiento del niño en casa y en la escuela, dinámica familiar, contexto cultural, historia familiar de no conformidad de género, vida social del niño y seguridad infantil. Se debe poner atención a la evaluación psicosocial adecuada para la edad del niño. Cabe al equipo de Atención Primaria a la Salud aclarar las principales dudas de las familias, realizar la sospechosa diagnóstica y encaminar al centro especializado de atención a los niños transgéneros de su estado. Conclusión: Cabe al equipo de Atención Primaria a la Salud proporcionar un ambiente acogedor y sin discriminación para que estas familias sean evaluadas y conducidas de forma adecuada.

Palabras clave: Identidad de Género; Género y Salud; Personas Transgénero; Disforia de Género; Niño

\section{Introdução}

A Disforia de Gênero se refere ao estresse que pode vir acompanhado de incongruência entre o gênero experimentado ou expressado e o gênero designado. ${ }^{1} \mathrm{O}$ gênero designado é aquele estabelecido ao nascimento pela identificação da genitália externa (sexo masculino ou feminino), já o gênero experimentado ou expressado é aquele que engloba sentimentos e identificação pessoal que não se restringem ao estereótipo binário homem-mulher. ${ }^{2}$ Estudos conduzidos em centros especializados no atendimento a crianças transgêneras evidenciam um aumento da procura por atendimento que pode ser devido ao crescente interesse pelo tema Disforia de Gênero e não conformidade de gênero. ${ }^{3-7}$ Segundo a quinta edição do Diagnostic and Statistical Manual of Mental Disorders (DSM-V), ${ }^{1}$ a prevalência de Disforia de Gênero entre homens adultos varia de $0,005 \%$ a $0,014 \%$, e em mulheres adultas $0,002 \%$ a $0,003 \%$, e a relação por sexo em crianças varia de 2:1 a 4,5:1 em meninos e meninas, respectivamente.

Pesquisadores identificaram que, aos 12 meses de vida, as crianças são capazes de determinar objetos típicos de cada gênero e, entre 17 e 21 meses de vida, elas se autoidentificam como meninos ou meninas; assim, a identidade de gênero tem início entre 2 e 3 anos de idade..$^{1-3}$ Para algumas crianças pré-escolares, a não conformidade de gênero, desejar pertencer a outro gênero ou, mais raramente, se rotular como membro de outro gênero, pode ocorrer devido ao seu desenvolvimento natural e exploração da sua individualidade. ${ }^{1}$ Por volta dos 6 a 7 anos de idade, a criança tem consciência de que seu gênero permanecerá o mesmo, ${ }^{2}$ e expressões de disforia anatômica podem ocorrer tanto por antecipação da puberdade quanto por Disforia de Gênero. ${ }^{1}$ 
Cabe à equipe de Atenção Primária à Saúde esclarecer as principais dúvidas das famílias, realizar a suspeita diagnóstica e encaminhar ao centro especializado no atendimento a crianças transgêneras do seu estado. ${ }^{2,3,8} \mathrm{O}$ objetivo desta pesquisa foi realizar revisão integrativa de pesquisas sobre Disforia de Gênero em crianças e identificar recomendações para o manejo na Atenção Primária à Saúde.

\section{Métodos}

Foi realizada revisão integrativa da literatura em 23 de novembro de 2018 utilizando os termos MeSH Gender Identity, Transgender Persons, Gender Dysphoria, Health Services for Transgender Persons, Children e Adolescent nas bases de dados PubMed, Medline, Lilacs, Scielo e busca manual on-line para artigos publicados entre 2008 e 2018. Como critério de inclusão, estabeleceu-se estudos que utilizassem crianças transgêneras. Foram excluídos artigos que abordassem exclusivamente: crianças transgêneras com Distúrbio da Diferenciação do Sexo, pais ou responsáveis de crianças transgêneras, adolescentes transgêneros, tratamento endocrinológico e/ou psiquiátrico para pessoas transgêneras. Com base nos artigos selecionados, foram identificadas recomendações para o manejo das famílias e crianças com Disforia de Gênero na Atenção Primária à Saúde.

\section{Resultados}

Dos 2.488 artigos identificados pela chave de busca, 277 foram selecionados pelo título, 83 pelo resumo, 31 pela leitura do texto completo e 12 artigos selecionados, conforme Figura 1.

Os artigos selecionados foram em sua maioria de amostras de centros especializados no atendimento a crianças transgêneras no Canadá, Estados Unidos, Inglaterra e Holanda. Os principais resultados do Quadro 1 limitaram-se aos dados de crianças menores de 12 anos de idade apresentados pelas pesquisas.

\section{Epidemiologia}

Um estudo de base populacional em Tóquio ${ }^{16}$ evidenciou Disforia de Gênero em 0,5\% dos meninos e 1,6\% das meninas entre 3 e 12 anos de idade. Contudo, devido ao instrumento de pesquisa ter sido questionário autoaplicável, os respondentes do grupo entre 3 e 12 anos de idade foram seus responsáveis, o que pode acarretar um viés de pesquisa. A prevalência de Disforia de Gênero em crianças ainda não foi estabelecida, pois entre as pesquisas que estimam este valor temos como respondentes os responsáveis pelas crianças e critérios diagnósticos desatualizados. Entretanto, estima-se que a prevalência de Disforia de Gênero em crianças seja menor que $1 \% .^{5,21}$

Os centros especializados no atendimento a pessoas com não conformidade de gênero e transgêneras, citados na pesquisa, assistiam às populações adulta e pediátrica. Na presente pesquisa, o percentual de crianças pré-púberes em atendimento nos centros especializados variou entre $7 \%$ e $42 \%{ }^{11,13,19,20} \mathrm{com}$ predomínio do sexo masculino. ${ }^{11}$ Em Toronto, a prevalência de Disforia de Gênero em menores de 12 anos de idade referenciadas para acompanhamento em centros especializados variou entre $12 \%{ }^{9}$ e $50 \%{ }^{14}(82 \%$ do sexo masculino ${ }^{14}$ ); já em Amsterdam foi de $75 \% .{ }^{10} \mathrm{Um}$ estudo de coorte de crianças com não conformidade de gênero de base populacional nos Estados Unidos, TransYouth Project, destacou que a proporção das faixas etárias de crianças em acompanhamento foram de $30 \%$ entre 3 e 5 anos de idade, $40 \%$ entre 6 e 8 anos de idade e $30 \%$ entre 9 e 12 anos de idade, com $70 \%$ de participação de crianças do sexo masculino. ${ }^{15}$ 


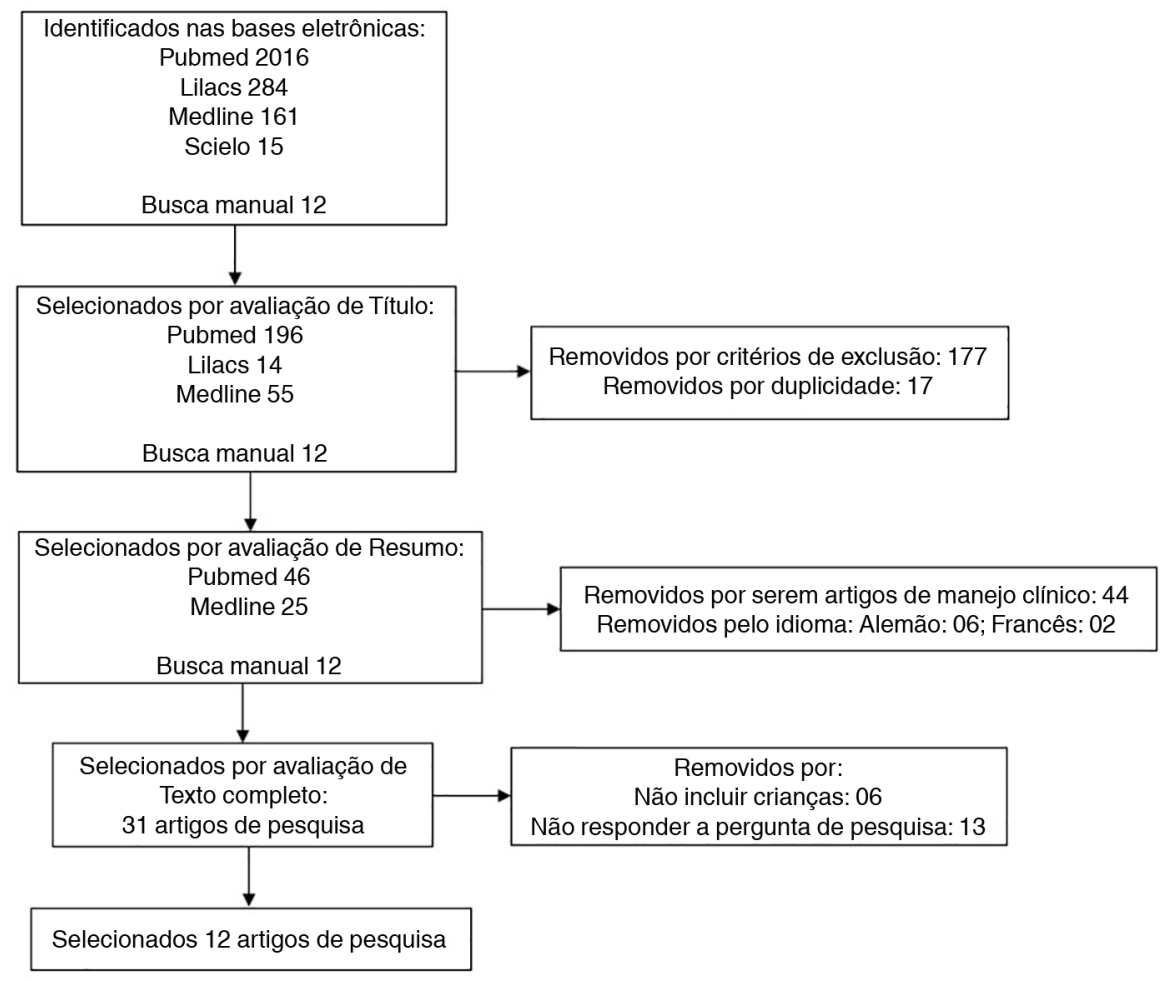

Figura 1. Fluxograma das etapas de seleção dos artigos de pesquisa sobre crianças transgêneras.

Quadro 1. Características dos artigos selecionados.

\begin{tabular}{|c|c|c|c|c|c|}
\hline Autores (ano) & $\begin{array}{l}\text { Tipo de } \\
\text { estudo }\end{array}$ & $\begin{array}{l}\text { Local } \\
\text { (período) }\end{array}$ & Dados da pesquisa & Principais resultados & Conclusão \\
\hline $\begin{array}{l}\text { Drummond et al. } \\
(2008)^{9}\end{array}$ & Coorte & $\begin{array}{l}\text { Toronto, } \\
\text { Canadá } \\
(1977-2002)\end{array}$ & $\begin{array}{l}\text { Crianças referenciadas ao } \\
\text { centro especializado } \\
25 \text { meninas participaram do } \\
\text { estudo } \\
\text { Idades entre } 3 \text { e } 12 \text { anos (inter- } \\
\text { valo para } 2^{\circ} \text { coleta: } 3 \text { a } 27 \text { anos) }\end{array}$ & $\begin{array}{l}\text { 10 avaliação: } 88 \% \text { meninas com } \\
\text { Desordem de Identidade de } \\
\text { gênero e } 12 \% \text { com Disforia de } \\
\text { gênero } \\
2^{\circ} \text { avaliação: } 60 \% \text { permanece- } \\
\text { ram Desordem de Identidade de } \\
\text { Gênero; } 28 \% \text { se identificaram } \\
\text { como homossexuais, bissexuais } \\
\text { ou assexuais }\end{array}$ & $\begin{array}{l}\text { Entre as participantes, houve } \\
\text { maior probabilidade de Disforia } \\
\text { de Gênero e Identidade Sexual } \\
\text { não heterossexual do que na } \\
\text { população em geral }\end{array}$ \\
\hline $\begin{array}{l}\text { Wallien \& } \\
\text { Cohen-Kettenis } \\
(2008)^{10}\end{array}$ & Coorte & $\begin{array}{l}\text { Amsterdam, } \\
\text { Holanda } \\
(1989-2005)\end{array}$ & $\begin{array}{l}\text { Crianças referenciadas ao } \\
\text { centro especializado } \\
77 \text { crianças participaram do } \\
\text { estudo entre } 5 \text { e } 12 \text { anos } \\
54 \text { crianças participaram do } \\
\text { seguimento entre } 16 \text { e } 28 \text { anos }\end{array}$ & $\begin{array}{l}\text { 10 avaliação: } 75 \% \text { com Disforia } \\
\text { de Gênero } \\
\text { 20 avaliação: } 27 \% \text { persistiram } \\
\text { com Disforia de Gênero (1 a cada } \\
5 \text { meninos; } 1 \text { a cada } 2 \text { meninas) } \\
\text { Entre os } 21 \text { participantes (todos } \\
\text { com Disforia de Gênero na } \\
\text { infância) houve maior não con- } \\
\text { formidade de gênero, disforia } \\
\text { de gênero, insatisfação corporal } \\
\text { (especificamente características } \\
\text { sexuais secundárias) em com- } \\
\text { paração ao grupo desistente } \\
68 \% \text { do grupo com Disforia de } \\
\text { Gênero relatou atração pelo } \\
\text { mesmo sexo e } 81 \% \text { se iden- } \\
\text { tificaram como bissexuais ou } \\
\text { homossexuais. }\end{array}$ & $\begin{array}{l}\text { A maioria das crianças com } \\
\text { Disforia de Gênero não perma- } \\
\text { necerá após a puberdade. } \\
\text { As crianças participantes que } \\
\text { persistiram com Disforia de } \\
\text { Gênero na adolescência apre- } \\
\text { sentaram Disforia de Gênero } \\
\text { extremas na infância. } \\
\text { O desfecho mais provável da } \\
\text { Disforia de Gênero na infância } \\
\text { foi homossexualidade ou bisse- } \\
\text { xualidade. }\end{array}$ \\
\hline
\end{tabular}


Continuação Quadro 1.

\begin{tabular}{|c|c|c|c|c|c|}
\hline Autores (ano) & $\begin{array}{l}\text { Tipo de } \\
\text { estudo }\end{array}$ & $\begin{array}{l}\text { Local } \\
\text { (período) }\end{array}$ & Dados da pesquisa & Principais resultados & Conclusão \\
\hline $\begin{array}{l}\text { Spack et al. } \\
(2012)^{11}\end{array}$ & Transversal & $\begin{array}{l}\text { Boston, Es- } \\
\text { tados Unidos } \\
(1998-2009)\end{array}$ & $\begin{array}{l}\text { Pessoas referenciadas ao cen- } \\
\text { tro especializado } \\
97 \text { participantes } \\
11 \text { em Estágio Tanner } 1 \text { ( } 7 \text { sexo } \\
\text { masculino); } 10 \text { Estágio Tanner } 2 \\
\text { ( } 5 \text { sexo masculino) }\end{array}$ & $\begin{array}{l}\text { Do total de participantes: } 45 \% \\
\text { demonstraram não conformida- } \\
\text { de de gênero antes dos } 5 \text { anos } \\
\text { de idade }\end{array}$ & $\begin{array}{l}\text { A importância do conhecimento } \\
\text { pelos profissionais de saúde } \\
\text { acerca da Desordem de Identi- } \\
\text { dade de Gênero para aconse- } \\
\text { Ihamento dos pais, prevenção } \\
\text { de automutilação e ideação } \\
\text { suicida, entre outras doenças } \\
\text { psiquiátricas }\end{array}$ \\
\hline $\begin{array}{l}\text { Olson et al. } \\
(2015)^{12}\end{array}$ & $\begin{array}{l}\text { Caso- } \\
\text { Controle }\end{array}$ & $\begin{array}{l}\text { Estados } \\
\text { Unidos (não } \\
\text { informado) }\end{array}$ & $\begin{array}{l}\text { Estudo coorte TransYouth } \\
\text { Project } \\
32 \text { (casos) crianças entre } 5 \text { e } 12 \\
\text { anos (pré-púberes) } 32 \text { controles } \\
\text { e } 18 \text { irmãos }\end{array}$ & $\begin{array}{l}\text { Não houve diferença signifi- } \\
\text { cativa segundo identidade de } \\
\text { gênero, preferências de gênero, } \\
\text { preferência de pares e objetos } \\
\text { preferidos entre os grupos }\end{array}$ & $\begin{array}{l}\text { O grupo de crianças transgêne- } \\
\text { ras que vivem de acordo com } \\
\text { sua identidade de gênero tem } \\
\text { sua autopercepção de acordo } \\
\text { com sua expressão de gênero }\end{array}$ \\
\hline $\begin{array}{l}\text { Hughes et al. } \\
(2016)^{14}\end{array}$ & Transversal & $\begin{array}{l}\text { Toronto, } \\
\text { Canadá } \\
(1976-2013)\end{array}$ & $\begin{array}{l}\text { Crianças referenciadas ao } \\
\text { centro especializado } \\
1.023 \text { casos (referenciadas } \\
\text { entre } 1976 \text { e 2013) } \\
1.064 \text { controles (referenciadas } \\
\text { a outras clínicas entre } 1991 \text { e } \\
2001 \text { ) }\end{array}$ & $\begin{array}{l}50 \% \text { dos casos referenciados } \\
\text { eram }<12 \text { anos com Disforia de } \\
\text { Gênero ( } 82 \% \text { sexo masculino) } \\
5 \% \text { dos casos referenciados } \\
\text { eram }<12 \text { anos com Fetichismo } \\
\text { Transvestista (todos sexo } \\
\text { masculino) }\end{array}$ & $\begin{array}{l}\text { Entre os casos homossexuais } \\
\text { (adolescentes) e de Fetichismo } \\
\text { Transvestista do sexo masculi- } \\
\text { no, houve menor probabilidade } \\
\text { de serem filhos únicos. Os } \\
\text { autores destacam o efeito de } \\
\text { ordem de nascimento fraternal } \\
\text { com maior probabilidade de } \\
\text { terem irmãos mais velhos }\end{array}$ \\
\hline $\begin{array}{l}\text { Sasaki et al. } \\
(2016)^{16}\end{array}$ & Transversal & $\begin{array}{l}\text { Tóquio, Japão } \\
\text { (não informa- } \\
\text { do) }\end{array}$ & $\begin{array}{l}\text { Estudo de base populacional } \\
\text { (Grupos de } 3-12 \text { anos; } 13-18 \\
\text { anos; } 19-26 \text { anos) } \\
2.202 \text { Gêmeos monozigóticos } \\
(1.074 ; 738 ; 390) \\
1.130 \text { Gêmeos dizigóticos } \\
(674 ; 302 ; 154) \\
\text { 1.022 Gêmeos dizigóticos } \\
\text { masc/fem (618;302;102) }\end{array}$ & $\begin{array}{l}\text { Prevalência de Disforia de } \\
\text { Gênero: grupo 3-12 anos (masc } \\
\text { 0,5\%, fem1,6\%); grupo 13-18 } \\
\text { anos (masc } 2 \% \text {, fem 10\%); } \\
\text { grupo } 19-26 \text { anos (masc 3\%, } \\
\text { fem 12\%) } \\
\text { Gêmeos dizigóticos masc/fem } \\
\text { não evidenciaram maiores } \\
\text { escores de Desordem de Identi- } \\
\text { dade de Gênero, portanto, este } \\
\text { achado não apoia a teoria de } \\
\text { transferência hormonal pré-natal } \\
\text { No sexo feminino } 84 \% \text { da vari- } \\
\text { ância na Desordem de Identi- } \\
\text { dade de Gênero pôde ser expli- } \\
\text { cada por fatores genéticos; no } \\
\text { sexo masculino } 70 \% \text { pôde ser } \\
\text { explicada por fatores ambientais } \\
\text { compartilhados }\end{array}$ & $\begin{array}{l}\text { A sociedade japonesa permite } \\
\text { que o sexo feminino possa ex- } \\
\text { pressar sua Disforia de Gênero } \\
\text { e não conformidade de gênero } \\
\text { mais amplamente do que o sexo } \\
\text { masculino } \\
\text { Os dados não corroboram a } \\
\text { teoria de transferência hormonal } \\
\text { pré-natal } \\
\text { A maior parte da variância para } \\
\text { Desordem de Identidade de } \\
\text { Gênero ocorreu por fatores fa- } \\
\text { miliares em crianças, entretanto, } \\
\text { esta variância parece diminuir } \\
\text { conforme a idade }\end{array}$ \\
\hline
\end{tabular}


Continuação Quadro 1.

\begin{tabular}{|c|c|c|c|c|c|}
\hline Autores (ano) & $\begin{array}{l}\text { Tipo de } \\
\text { estudo }\end{array}$ & $\begin{array}{l}\text { Local } \\
\text { (período) }\end{array}$ & Dados da pesquisa & Principais resultados & Conclusão \\
\hline $\begin{array}{l}\text { Drummond et al. } \\
(2017)^{17}\end{array}$ & Coorte & $\begin{array}{l}\text { Toronto, } \\
\text { Canadá } \\
(1977-2002)\end{array}$ & $\begin{array}{l}\text { Crianças referenciadas ao } \\
\text { centro especializado } \\
24 \text { meninas participaram do } \\
\text { estudo } \\
\text { Idades entre } 3 \text { e } 12 \text { anos (inter- } \\
\text { valo para } 2^{2} \text { coleta: } 3 \text { a } 27 \text { anos) }\end{array}$ & $\begin{array}{l}\text { 1ª avaliação: } 45 \% \text { dos casos } \\
\text { apresentaram problemas de } \\
\text { comportamento; } 46 \% \text { apresen- } \\
\text { taram depressão ou ansiedade; } \\
42 \% \text { abuso ou dependência de } \\
\text { substâncias } \\
2^{\text {a }} \text { avaliação: } 39 \% \text { dos res- } \\
\text { ponsáveis e } 33 \% \text { dos casos } \\
\text { auto relataram problemas de } \\
\text { comportamento; } 33 \% \text { relataram } \\
\text { duas ou mais tentativas de } \\
\text { suicídio desde } 13 \text { anos }\end{array}$ & $\begin{array}{l}\text { Meninas referenciadas mostra- } \\
\text { ram vulnerabilidade psiquiátrica } \\
\text { no seguimento e destaca a } \\
\text { importância de abordagem inte- } \\
\text { grativa e holística nos cuidados } \\
\text { destes pacientes }\end{array}$ \\
\hline $\begin{array}{l}\text { Durwood et al. } \\
(2017)^{18}\end{array}$ & $\begin{array}{l}\text { Caso- } \\
\text { Controle }\end{array}$ & $\begin{array}{l}\text { Estados } \\
\text { Unidos (2015- } \\
\text { 2016) }\end{array}$ & $\begin{array}{l}\text { Estudo coorte Trans Youth } \\
\text { Project } \\
63 \text { (casos) crianças entre 9-14 } \\
\text { anos } 63 \text { controles e } 38 \text { irmãos } \\
116 \text { (casos) crianças entre 6-14 } \\
\text { anos } 122 \text { controles e } 72 \text { irmãos }\end{array}$ & $\begin{array}{l}\text { No grupo de 9-14 anos houve } \\
\text { taxas normais de depressão e } \\
\text { taxas pouco aumentadas de } \\
\text { ansiedade } \\
\text { Os responsáveis do grupo de } \\
\text { 9-14 anos não apresentaram } \\
\text { diferença nas taxas de depres- } \\
\text { são entre os grupos, porém } \\
\text { apresentaram taxas altas de } \\
\text { ansiedade no grupo de casos } \\
\text { No grupo de 6-14 anos houve } \\
\text { alta autoestima global que não } \\
\text { diferenciou entre os grupos }\end{array}$ & $\begin{array}{l}\text { Ser transgênero não é sinônimo } \\
\text { de altos níveis de psicopatias } \\
\text { e fornece evidências conver- } \\
\text { gentes de que o apoio familiar } \\
\text { precoce está associado a uma } \\
\text { saúde mental positiva em crian- } \\
\text { ças transgêneras }\end{array}$ \\
\hline $\begin{array}{l}\text { Lawlis et al. } \\
(2017)^{19}\end{array}$ & Transversal & $\begin{array}{l}\text { Ohio, Estados } \\
\text { Unidos (não } \\
\text { informado) }\end{array}$ & $\begin{array}{l}\text { Pessoas referenciadas ao cen- } \\
\text { tro especializado } \\
118 \text { casos participantes; } 103 \\
\text { responsáveis } \\
8 \text { entre } 6 \text { e } 10 \text { anos; } 24 \text { entre } 11 \\
\text { e } 14 \text { anos }\end{array}$ & $\begin{array}{l}\text { No grupo de casos de 6-10 } \\
\text { anos as maiores preocupações } \\
\text { foram uso de banheiros, bloque- } \\
\text { adores de puberdade e senti- } \\
\text { mento de segurança na escola } \\
\text { Os responsáveis do grupo de } \\
6-10 \text { anos apresentaram maio- } \\
\text { res preocupações com aceita- } \\
\text { ção familiar, uso de banheiros, } \\
\text { recursos gerais, questões legais } \\
\text { e discutir questões trans com } \\
\text { membros não familiares }\end{array}$ & $\begin{array}{l}\text { O grupo 6-10 anos possi- } \\
\text { velmente não compreende } \\
\text { as questões futuras a serem } \\
\text { enfrentadas por pessoas trans- } \\
\text { gêneras e apresentaram pouca } \\
\text { preocupação com a transição } \\
\text { física visto que estão em fase } \\
\text { pré-púbere. } \\
\text { Os responsáveis deste grupo } \\
\text { estiveram mais preocupados } \\
\text { com questões sociais do que } \\
\text { clínicas }\end{array}$ \\
\hline $\begin{array}{l}\text { Wiepjes et al. } \\
(2018)^{20}\end{array}$ & Transversal & $\begin{array}{l}\text { Amsterdam, } \\
\text { Holanda } \\
(1972-2015)\end{array}$ & $\begin{array}{l}\text { Pessoas referenciadas ao cen- } \\
\text { tro especializado } \\
6.793 \text { participantes } \\
548 \text { crianças entre } 7-10 \text { anos } \\
\text { de idade }\end{array}$ & $\begin{array}{l}\text { Em 2015, prevalência } 1 \text { mulher } \\
\text { trans: } 3.800 \text { homens; } 1 \text { homem } \\
\text { trans: } 5.200 \text { mulheres } \\
8 \% \text { de crianças entre } 7-10 \text { anos } \\
\text { de idade referenciadas }\end{array}$ & $\begin{array}{l}\text { Houve aumento dramático da } \\
\text { procura por atendimento espe- } \\
\text { cializado nas últimas décadas }\end{array}$ \\
\hline
\end{tabular}

O diagnóstico de Disforia de Gênero requer sucessivas avaliações, pois pode ser confundido com comportamentos próprios da exploração da identidade de gênero da criança. Hughes et al. ${ }^{14}$ destacaram que $5 \%$ das crianças menores de 12 anos de idade referenciadas apresentaram Fetichismo Transvestista (todas do sexo masculino), e na coorte de Spack et al. ${ }^{11} 45 \%$ dos participantes relataram não conformidade de gênero antes dos 5 anos de idade. No estudo de Wallien \& Cohen-Kettenis ${ }^{10}$ foi observado que todos os participantes com Disforia de Gênero permaneceram com não conformidade de gênero, Disforia de Gênero e insatisfação corporal (especificamente para caracteres sexuais secundários) no seguimento. 
Nem toda criança que apresenta não conformidade de gênero ou Disforia de Gênero nas primeiras avaliações permanecerá com este diagnóstico no seguimento ao longo dos anos. Dois estudos de coorte publicados em 2008 mostraram dados do seguimento dos participantes com $60 \%$ de persistência de não conformidade de gênero ${ }^{9}$ e $27 \%$ de Disforia de Gênero ${ }^{10}$ (1:5 meninos; $1: 2$ meninas). ${ }^{10}$ Nestes mesmos estudos, os já adolescentes se autoidentificaram como homossexuais ou bissexuais em $28 \%{ }^{9}$ e $81 \%{ }^{10}$

A etiologia e fatores influenciadores da Disforia de Gênero ainda demandam estudos da comunidade científica. Sasaki et al. ${ }^{16}$ em sua pesquisa com gêmeos, destacou que os dados sobre gêmeos dizigóticos masculino/feminino não corroboraram a teoria de transferência hormonal pré-natal e que $84 \%$ da variância da Desordem de Identidade de Gênero pôde ser explicada por fatores genéticos no sexo feminino, o que não ocorreu no sexo masculino, onde a variância foi explicada em $70 \%$ por fatores ambientais.

Outro interesse dos pesquisadores foi a vulnerabilidade emocional destas crianças. Dados do TransYouth Project mostraram que crianças que viveram de acordo com a sua identidade de gênero (transição social) tinham sua autopercepção em acordo com sua expressão de gênero ${ }^{12}$ e apresentaram sua autoestima global elevada. ${ }^{18}$ As crianças que realizaram a transição social com apoio familiar evidenciaram taxas típicas de depressão para a idade e pouco elevadas de ansiedade. ${ }^{15}$ Já os pais destas crianças apresentaram altas taxas de ansiedade. ${ }^{18} \mathrm{Em}$ outro estudo, não foi especificado se houve transição social ou apoio familiar, mas na primeira avaliação crianças entre 3 e 12 anos de idade apresentaram distúrbios de comportamento em $45 \%$ da amostra, depressão ou ansiedade em $46 \%$ e abuso ou dependência de substâncias em 42\%; no seguimento após no mínimo 3 anos da primeira avaliação, 33\% apresentaram distúrbios de comportamento, e 33\% relataram duas ou mais tentativas de suicídio antes dos 13 anos de idade. ${ }^{17}$

A aceitação da sociedade para crianças com não conformidade de gênero ou Disforia de Gênero reflete nas pesquisas sobre o tema. Em Londres, crianças menores de 12 anos referenciadas ao centro especializado relataram bullying, ansiedade, automutilação e ideação suicida. ${ }^{13}$ Outra pesquisa evidenciou que suas maiores preocupações foram uso de banheiros e vestiários, bloqueadores de puberdade e segurança na escola. Seus pais reportaram preocupações com a aceitação familiar, uso de banheiros e vestiários, recursos gerais, questões legais e discutir questões trans com membros não familiares. ${ }^{19}$

\section{Avaliação e critérios diagnósticos}

A equipe da Atenção Primária à Saúde que recebe uma queixa ou dúvida sobre o comportamento ou identidade de gênero de uma criança deve acolher a família, ouvir suas demandas e observar a criança. Criar um ambiente seguro de aceitação sem julgamentos que possam prejudicar e/ou discriminar esta família.,22 O profissional deve realizar anamnese direcionada às questões de comportamento da criança em casa e na escola, dinâmica familiar, contexto cultural, história familiar de não conformidade de gênero, vida social da criança e segurança infantil. ${ }^{22,23}$ Deve-se atentar para a avaliação psicossocial adequada para a idade da criança. ${ }^{23}$ Conforme evidenciado, estas crianças estão sujeitas a situações de vulnerabilidade e podem apresentar depressão, ansiedade, automutilação e ideação suicida por não compreensão da sua identidade de gênero, bullying ou não aceitação familiar. ${ }^{13,17,19}$ 
Posteriormente, realizar exame físico geral e específico para Distúrbios de Diferenciação do Sexo e sinais de Puberdade Precoce. ${ }^{3}$ Estas são situações que devem ser avaliadas minuciosamente pelo médico responsável e realizada a condução clínica recomendada para cada situação. Não há indicação de exames laboratoriais na avaliação de Disforia de Gênero em crianças, exceto na suspeita de Distúrbios da Diferenciação do Sexo e/ou Puberdade Precoce. ${ }^{3}$

Realizada a suspeita diagnóstica, esta família deve ser encaminhada ao centro especializado no acompanhamento a crianças transgêneras do seu estado, que realizará o acompanhamento por pelo menos 6 meses (fase de diagnóstico) ${ }^{8}$ no qual serão verificados os seguintes critérios (Quadro 2):

Quadro 2. Critérios diagnósticos para Disforia de Gênero em crianças.

Critérios diagnósticos para Disforia de Gênero em crianças

A. Incongruência acentuada entre o gênero experimentado/expresso e o gênero designado de uma pessoa, com duração de pelo menos seis meses, manifestada por, no mínimo, seis dos seguintes oito critérios (um deles deve ser o critério A1):

1. Forte desejo de pertencer ao outro gênero ou insistência de que um gênero é o outro (ou algum gênero alternativo diferente do designado)

2. Em meninos (gênero designado), uma forte preferência por cross-dressing (travestismo) ou simulação de trajes femininos; em meninas (gênero designado), uma forte preferência por vestir somente roupas masculinas típicas e uma forte resistência a vestir roupas femininas típicas

3. Forte preferência por papéis transgêneros em brincadeiras de faz de conta ou de fantasias

4. Forte preferência por brinquedos, jogos ou atividades tipicamente usados ou preferidos por outro gênero.

5. Forte preferência por brincar com pares do outro gênero.

6. Em meninos (gênero designado), forte rejeição de brinquedos, jogos ou atividades tipicamente masculinas e forte evitação de brincadeiras agressivas e competitivas; em meninas (gênero designado), forte rejeição de brinquedos, jogos e atividades tipicamente femininas.

7. Forte desgosto com a própria anatomia sexual.

8. Desejo intenso por características sexuais primárias e/ou secundárias compatíveis com o gênero experimentado.

B. A condição está associada a sofrimento clinicamente significativo ou a prejuízo no funcionamento social, acadêmico ou em outras áreas importantes da vida do indivíduo.

Especificar se:

Com um Distúrbio da Diferenciação do Sexo

Fonte: Adaptado de American Psychiatric Association ${ }^{1}$ e Sociedade Brasileira de Pediatria. ${ }^{2}$

O diagnóstico definitivo é um desafio e demanda equipe multidisciplinar experiente nestas situações, pois são necessárias intervenções psicoterápicas para a criança, família e em grupo. ${ }^{21,22}$

\section{Recomendações}

O objetivo do trabalho da equipe de Atenção Primária à Saúde é proporcionar o desenvolvimento do bem-estar físico, emocional e social destas famílias. ${ }^{21,22} \mathrm{~A}$ vergonha e o medo da estigmatização podem tardar a procura por atendimento, por isso a equipe deve ser respeitosa e livre de julgamentos..$^{21,23}$ Estudos evidenciam que crianças com comportamentos de não conformidade de gênero ou Disforia de Gênero, poderão se identificar como homossexuais ou bissexuais na adolescência. ${ }^{9,10}$ 
A principal preocupação deve ser identificar riscos para a segurança da criança no seu ambiente familiar e social, de forma a protegê-la, para que ela possa explorar sua identidade de gênero. ${ }^{3,8}$ Como evidenciado nas pesquisas, quando há apoio familiar estas crianças apresentam alta autoestima global e sentimentos favoráveis sobre seu desenvolvimento. ${ }^{12,15,18}$ A confidencialidade das informações compartilhadas pela família deve ter especial consideração. ${ }^{23}$

As considerações éticas e a necessidade de acompanhamento a longo prazo sinalizam que o médico de Família e Comunidade deve sempre buscar equipe multidisciplinar para o manejo destas famílias e crianças. ${ }^{2} \mathrm{O}$ acolhimento deve ser individualizado e o acompanhamento de forma integral. ${ }^{2}$ É $^{-}$ responsabilidade do médico identificar se a criança preenche os critérios diagnósticos, avaliar suporte social, saúde mental e se há interesse da criança e da família em realizar intervenções clínicas. ${ }^{2}$ Com a suspeita diagnóstica, esta família deve ser encaminhada ao centro especializado de referência.

Quando a criança é referenciada, ela é acompanhada por, no mínimo, 6 meses com psicoterapia que tem como foco a identidade de gênero, apoio social, imagem corporal, promoção da resiliência e suporte a problemas psíquicos. ${ }^{1,2,4,7,8} \mathrm{~A}$ fase seguinte pode durar de 3 a 4 anos e evolve a supressão puberal. Esta é iniciada no estágio 2 de Tanner com o objetivo de suprimir a produção dos esteroides sexuais, e assim, as modificações físicas da puberdade. .,2,7,8 $^{-1,0}$

Os medicamentos usados são os agonistas do hormônio liberador de gonadotrofina, e medroxiprogesterona como tratamento alternativo. ${ }^{2}$ Esta intervenção é reversível com desenvolvimento puberal imediato após suspensão da medicação. ${ }^{2,8}$ A hormonioterapia ocorre no adolescente acima de 16 anos de idade com consentimento dos pais sincronizada com a equipe especializada. ${ }^{1,2,8,24}$ Nesta fase, a terapia hormonal envolve masculinizar ou feminilizar o corpo do adolescente com testosterona ou estrógenos, respectivamente. ${ }^{1,2,8} \mathrm{O}$ tratamento cirúrgico é um recurso para adultos..$^{1,2,8}$

O uso de bloqueadores da puberdade ou hormonioterapia somente são indicados em casos de certeza do diagnóstico de Disforia de Gênero e seguem recomendações do parecer do Conselho Federal de Medicina $n^{\circ} 8 / 2013 .{ }^{24}$

Como desafios temos a falta de acesso aos serviços especializados, pois se localizam em grandes centros urbanos, a crítica social na abordagem destes casos, burocracia, demora no atendimento nos centros especializados e falta de treinamento das equipes na abordagem destas situações. ${ }^{22}$

\section{Conclusão}

O médico de Família e Comunidade deve realizar a suspeita diagnóstica por meio da investigação clínica por anamnese e exame físico. Assegurar um ambiente acolhedor e profissional sem julgamentos ou discriminação para que a família possa se sentir segura ao expor suas dúvidas. A principal preocupação é a identificação de potenciais riscos à segurança da família e da criança no seu contexto social e cultural. Deve-se garantir o bem-estar físico, emocional e social desta criança para que ela possa desenvolver sua identidade de gênero adequadamente. O diagnóstico definitivo é responsabilidade de equipe multidisciplinar experiente em crianças com Disforia de Gênero. 


\section{Referências}

1. American Psychiatric Association - APA. Diagnostic and Statistical Manual of Mental Disorders: DSM -5. 5th ed. Washington: American Psychiatric Association; 2013.

2. Sociedade Brasileira de Pediatria. Departamento Científico de Adolescência. Guia Prático de Atualização: Disforia de Gênero [Internet]; 2017. [acesso 2018 Nov 30]. Disponível em: goo.gl/dfMHEz

3. Bidwell RJ. Gender expression and identity issues. In: Adam HM, Foy JM, eds.. Signs \& Symptoms in Pediatrics. Itasca: American Academy of Pediatrics; 2015. p. 409-42.

4. Ristori J, Steensma TD. Gender dysphoria in childhood. Int Rev Psychiatry. 2016;28(1):13-20. http://dx.doi.org/10.3109/09540261.2015.111 5754

5. Zucker KJ. Epidemiology of gender dysphoria and transgender identity. Sex Health. 2017;14(5):404-11. http://dx.doi.org/10.1071/SH17067

6. de Vries AL, Cohen-Kettenis PT. Clinical management of gender dysphoria in children and adolescents: the Dutch approach. J Homosex. 2012;59(3):301-20. http://dx.doi.org/10.1080/00918369.2012.653300

7. Butler G, De Graaf N, Wren B, Carmichael P. Assessment and support of children and adolescents with gender dysphoria. Arch Dis Child. 2018;103(7):631-6. http://dx.doi.org/10.1136/archdischild-2018-314992

8. de Vries AL, Klink D, Cohen-Kettenis PT. What the Primary Care Pediatrician Needs to Know About Gender Incongruence and Gender Dysphoria in Children and Adolescents. Pediatr Clin North Am. 2016;63(6):1121-35. http://dx.doi.org/10.1016/j.pcl.2016.07.011

9. Drummond KD, Bradley SJ, Peterson-Badali M, Zucker KJ. A follow-up study of girls with gender identity disorder. Dev Psychol. 2008;44(1):34-45. http://dx.doi.org/10.1037/0012-1649.44.1.34

10. Wallien MS, Cohen-Kettenis PT. Psychosexual outcome of gender-dysphoric children. J Am Acad Child Adolesc Psychiatry. 2008;47(12):1413-23. http://dx.doi.org/10.1097/CHI.0b013e31818956b9

11.Spack NP, Edwards-Leeper L, Feldman HA, Leibowitz S, Mandel F, Diamond DA, et al. Children and adolescents with gender identity disorder referred to a pediatric medical center. Pediatrics. 2012;129(3):418-25. http://dx.doi.org/10.1542/peds.2011-0907

12. Olson KR, Key AC, Eaton NR. Gender cognition in transgender children. Psychol Sci. 2015;26(4):467-74. http://dx.doi. org/10.1177/0956797614568156

13. Holt V, Skagerberg E, Dunsford M. Young people with features of gender dysphoria: Demographics and associated difficulties. Clin Child Psychol Psychiatry. 2016;21(1):108-18. http://dx.doi.org/10.1177/1359104514558431

14. Hughes SK, VanderLaan DP, Blanchard R, Wood H, Wasserman L, Zucker KJ. The Prevalence of Only-Child Status Among Children and Adolescents Referred to a Gender Identity Service Versus a Clinical Comparison Group. J Sex Marital Ther. 2017;43(6):586-93. http:// dx.doi.org/10.1080/0092623X.2016.1208702

15. Olson KR, Durwood L, DeMeules M, McLaughlin KA. Mental Health of Transgender Children Who Are Supported in Their Identities. Pediatrics. 2016;137(3):e20153223.

16. Sasaki S, Ozaki K, Yamagata S, Takahashi Y, Shikishima C, Kornacki T, et al. Genetic and Environmental Influences on Traits of Gender Identity Disorder: A Study of Japanese Twins Across Developmental Stages. Arch Sex Behav.2016;45(7):1681-95. http://dx.doi.org/10.1007/ s10508-016-0821-4

17. Drummond KD, Bradley SJ, Peterson-Badali M, VanderLaan DP, Zucker KJ. Behavior Problems and Psychiatric Diagnoses in Girls with Gender Identity Disorder: A Follow-Up Study. J Sex Marital Ther. 2018;44(2):172-87. http://dx.doi.org/10.1080/0092623X.2017.1340382

18. Durwood L, McLaughlin KA, Olson KR. Mental Health and Self-Worth in Socially Transitioned Transgender Youth. J Am Acad Child Adolesc Psychiatry. 2017;56(2):116-23.e2. http://dx.doi.org/10.1016/j.jaac.2016.10.016

19. Lawlis SM, Donkin HR, Bates JR, Britto MT, Conard LAE. Health Concerns of Transgender and Gender Nonconforming Youth and Their Parents Upon Presentation to a Transgender Clinic. J Adolesc Health. 2017;61(5):642-8. http://dx.doi.org/10.1016/j.jadohealth.2017.05.025

20. Wiepjes CM, Nota NM, de Blok CJM, Klaver M, de Vries ALC, Wensing-Kruger SA, et al. The Amsterdam Cohort of Gender Dysphoria Study (1972-2015): Trends in Prevalence, Treatment, and Regrets. J Sex Med. 2018;15(4):582-90. http://dx.doi.org/10.1016/j.jsxm.2018.01.016 
21. Puszczyk M, Czajeczny D. Gender dysphoria and gender variance in children - diagnostic and therapeutic controversies. Arch Psychiatr Psychother. 2017;19(3):34-42. http://dx.doi.org/10.12740/APP/74640

22. Torres CG, Renfrew M, Kenst K, Tan-McGrory A, Betancourt JR, López L. Improving transgender health by building safe clinical environments that promote existing resilience: Results from a qualitative analysis of providers. BMC Pediatr. 2015;15:187. http://dx.doi. org/10.1186/s12887-015-0505-6

23. Adelson SL; American Academy of Child and Adolescent Psychiatry (AACAP) Committee on Quality Issues (CQI). Practice parameter on gay, lesbian, or bisexual sexual orientation, gender nonconformity, and gender discordance in children and adolescents. J Am Acad Child Adolesc Psychiatry. 2012;51(9):957-74. http://dx.doi.org/10.1016/j.jaac.2012.07.004

24. Brasil. Conselho Federal de Medicina - CFM. Parecer n`8/2013 [Internet]. 2013. [acesso 2018 Dez 23]. Disponível em: goo.gl/mgVHH8 